\section{Four-years experience with the preferential derivation care model for multidisciplinary care in patients with psoriasis and psoriatic arthritis}

\author{
Martina Burlando, ${ }^{1}$ Chiara Craviotto, ${ }^{2}$ \\ Luca Carmisciano, ${ }^{3}$ Gerolamo Bianchi, ${ }^{2}$ \\ Aurora Parodi ${ }^{1}$ \\ ${ }^{1}$ Division of Dermatology, Department of \\ Health and Science, Policlinico San \\ Martino Hospital, Genoa; ${ }^{2}$ Department \\ of Rheumatology, Asl 3 Genovese, \\ Genoa; ${ }^{3}$ Division of Biostatistics, \\ Department of Health Sciences, \\ Policlinico San Martino Hospital, \\ Genoa, Italy
}

Psoriatic arthritis (PsA) is a chronic disease in which both dermatologists and rheumatologists are involved. Nowadays, structured, multidisciplinary care is highly recommended by national and international societies and desired by patients. ${ }^{1,2}$ Three main ways of action have been proposed:3,4 a) multidisciplinary care unit; b) parallel multidisciplinary care model; c) preferential derivation care model. In the first one, patients are evaluated simultaneously by a team composed of a dermatologist and a rheumatologist. In the second model, patients are first examined by one of the specialists and immediately afterwards by the other one, in the same hospital. In the third one, following a predefined referral protocol, patients are examined independently by the professionals, who may also visit in different hospitals. The importance of using a multidisciplinary model to improve the management of patients with psoriasis or PsA has been highlighted. ${ }^{4}$ However, little is known about self-reported patients' benefits.

We herein report our 4 year-experience in the Dermatology Unit of the Policlinico San Martino Hospital in Genoa, using the preferential derivation care model. Aim of the present observational, descriptive study was to explore the benefits of a multidisciplinary care model in psoriatic and psoriatic arthritis patients.

Since 2016, all patients referring to our Dermatology clinic who fulfilled the following inclusion criteria were enrolled: a) aged 18 years or older, b) confirmed diagnosis of chronic plaque psoriasis and musculoskeletal symptoms; c) signed informed consent. Patients were excluded from the study if they already had a diagnosis of psoriatic arthritis.

Patients were asked to fill in the
Screening Tool for Rheumatologic Investigation in Psoriatic Patients (STRIPP) questionnaire. $^{5}$ Specifically, the STRIPP questionnaire was used as a screening test. ${ }^{5}$

All patients evaluated in the Dermatology clinic, with aSTRIPP score of more than 3, were sent to the rheumatologist within 7 days. Likewise, all patients evaluated in the Rheumatology Unit who had a diagnosis of seronegative arthritis, and any dermatological disease were sent to the dermatologist within 7 days. A database was created for data collection

All patients were asked to fill in the Short Assessment for Patient Satisfaction measure questionnaire (SAPS), translated into the local language. ${ }^{6}$ This tool is composed of 7 descriptive items that cover all patient satisfaction dimensions. Patients rated their satisfaction for each item from 0 (very satisfied) to 4 (very dissatisfied). Continuous variable age was reported by mean and standard deviation (SD). Data on categorical variables were reported by count and percentages.

The present study was conducted following the Helsinki Declaration of 1975 , as revised in 1983, on Ethical Principles for Medical Research Involving Human Subjects and approved by San Martino Ethical Committee with the number of 180

Over a period of 4 years (2016-2020) $\mathrm{n}=160$ patients $(\mathrm{men}=109$; women $=51$; mean age $=67 \pm 10$ ) were included, and $n=250$ visits were carried out. Sixty-eight $\%(n=170)$ of all visits were first visits, while $32 \%(n=80)$ were follow-up visits (of which $10 \%$ were third or more visits). The rate of dropouts was $5 \%$.

Over the 4-year study period, an average of 30 to 35 patients were seen per year. The results are reported in Table 1 .

All patients filled in the SAPS questionnaire, the mean score was $20( \pm 2)$, 3 points up the mean SAPS score indicating a good rate of satisfaction. When answering the question: "Was the time you had with the doctor too short?", most of them (90/160) replied "not sure". All the patients $(100 \%)$ positively rated the short wait between the first visit and the second (less than 7 days), Still all of them would have preferred simultaneous evaluation, considered less time-consuming. Unfortunately, a simultaneous visit is not always feasible in many hospitals like ours. The patient pays a sum for each visit, therefore in a simultaneous visit, the patient should co-pay for two visits, which is not legally possible. Anyway, using this care model, 28 PsA patients were diagnosed as soon as they referred to the hospital and were able to start therapy immediately after diagnosis. The remaining patients $(42 / 70)$ had other
Correspondence: Martina Burlando, Department of Dermatology, IRCCS San Martino Polyclinic Hospital, Largo Rosanna Benzi X, 16132 Genoa, Italy.

Tel.: +39.010.5555761 - Fax: +39.010.5556641. E-mail: martina.burlando@unige.it

Key words: Psoriatic arthritis, Dermatology, Rheumatology.

Contributions: The authors contributed equally.

Conflict of interest: The authors declare no potential conflict of interest.

Funding: None.

Ethical approval and consent to participate: Written informed consent was obtained from the patient.

Availability of data and material: Data and materials can be obtained by contacting the corresponding author

Please cite this article as: Burlando $M$, Craviotto C, Carmisciano L, et al. Four-year experience with the preferential derivation care model for multidisciplinary care in patients with psoriasis and psoriatic arthritis. Dermatol Rep 2022;14:9111.

Received for publication: 21 February 2021. Accepted for publication: 10 September 2021

This work is licensed under a Creative Commons Attribution-NonCommercial 4.0 International License (CC BY-NC 4.0).

(C) Copyright: the Author(s), 2021

Licensee PAGEPress, Italy

Dermatology Reports 2022; 14:9111

doi:10.4081/dr.2021.9111

Publisher's note: All claims expressed in this article are solely those of the authors and do not necessarily represent those of their affiliated organizations, or those of the publisher, the editors and the reviewers. Any product that may be evaluated in this article or claim that may be made by its manufacturer is not guaranteed or endorsed by the publisher.

rheumatologic diseases that the rheumatologists diagnosed and treated as well.

Our study has some limitations, first its observational and descriptive methodology. Second, the STRIPP and SAPS questionnaires have not been validated in Italian population, so far. Despite these limitations, our findings indicate that even if multidisciplinary collaboration is not carried out the same day in the same clinic, it may be a good strategy for dealing with psoriatic arthritis. Spanish authors have published 
Table 1. Number of patients, diagnoses and SAPS score.

\begin{tabular}{|c|c|c|c|c|c|c|}
\hline Specialist & Diagnosis & Patients, n. & Patients, \% & Diagnoses, n. & Diagnoses, \% & SAPS score \\
\hline Rheumatologist & $\begin{array}{l}\text { Early psoriatic arthritis } \\
\text { Osteoarthritis } \\
\text { Fibromyalgia } \\
\text { Gout } \\
\text { Nonspecific musculoskeletal pain }\end{array}$ & $\begin{array}{l}28 \\
24 \\
11 \\
6 \\
1\end{array}$ & $\begin{array}{l}17.5 \\
15.0 \\
6.9 \\
3.8 \\
0.6\end{array}$ & 70 & $\begin{array}{c}40 \\
34 \\
16 \\
9 \\
1\end{array}$ & 21 \\
\hline Dermatologist & $\begin{array}{l}\text { Onychopathy } \\
\text { Seborrheic d. } \\
\text { Psoriasis } \\
\text { Methotrexate-telogen effluvium } \\
\text { Drug eruption }\end{array}$ & $\begin{array}{l}25 \\
20 \\
20 \\
15 \\
10\end{array}$ & $\begin{array}{l}15.6 \\
12.5 \\
12.5 \\
9.4 \\
6.2\end{array}$ & 90 & $\begin{array}{l}28 \\
22 \\
22 \\
17 \\
11\end{array}$ & 20 \\
\hline
\end{tabular}

several articles on this subject. ${ }^{4}$ They do not state which model is better. Stillall agree that whatever is the model used, itimproves the efficiency of the health care system and promotes collaboration between specialities, as observed in our study.

\section{References}

1. Gossec L, Baraliakos X, Kerschbaumer A, et al. EULAR recommendations for the management of psoriatic arthritis with pharmacological therapies: 2019 update. Ann Rheum Dis 2020;79:70012.

2. Betteridge N, Boehncke W-H, Bundy C, et al. Promoting patient-centred care in psoriatic arthritis: a multidisciplinary European perspective on improving the patient experience. J Eur Acad Dermatol Venereol 2016;30:576-85.

3. Queiro R, Coto P, Rodríguez J, et al. Multidisciplinary care models for patients with psoriatic arthritis. Reumatol Clin 2016;13:85-90.

4. Gratacós J, Luelmo J, Rodríguez J, et al. Standards of care and quality indicators for multidisciplinary care models for psoriatic arthritis in Spain. Rheumatol Int 2018;38:1115-24.

5. Burlando M, Cozzani E, Schiavetti I, et al. The STRIPP questionnaire (Screening Tool for Rheumatologic Investigation in Psoriatic Patients) as a new tool for the diagnosis of early psoriatic arthritis. G Ital Dermatol Venereol 2020;155:294-8.

6. Hawthorne G, Sansoni J, Hayes L, et al. Measuring patient satisfaction with health care treatment using the Short Assessment of Patient Satisfaction measure delivered superior and robust satisfaction estimates. J Clin Epidemiol 2014;67:527-37. 